\title{
NOTAS TAXONÓMICAS SOBRE EL GÉNERO CENTAUREA L. (ASTERACEAE) EN LA PENÍNSULA IBÉRICA. II. C. CASTELLANOIDES TALAVERA Y C. ARISTATA HOFFMANNS. \& LINK
}

\author{
Eusebio LÓPEZ1 y Juan Antonio DEVESA² \\ ${ }^{1}$ Departamento de Biología Vegetal, Ecología y Ciencias de la Tierra (Área de Botánica), Facultad \\ de Ciencias, Universidad de Extremadura, Avenida de Elvas s/n, 06071-Badajoz, España \\ ${ }^{2}$ Departamento de Botánica, Ecología y Fisiología Vegetal, Facultad de Ciencias, Universidad de \\ Córdoba, Campus de Rabanales, Edificio José Celestino Mutis, \\ ctra. de Madrid km. 396, 14071-Córdoba, España \\ Autor para correspondencia: bv1dealj@uco.es \\ Recibido el17 de julio de 2008, aceptado para su publicación el 2 de septiembre de 2008 \\ Publicado "on line" en octubre de 2009
}

\begin{abstract}
RESUMEN. Notas taxonómicas sobre el género Centaurea en la Península Ibérica. II. C. castellanoides Talavera y C. aristata Hoffmanns. \& Link (Asteraceae). Se comenta la variabilidad morfológica de $C$. castellanoides Talavera en la Península Ibérica, un endemismo para el que se reconocen tres subespecies, dos de ellas nuevas: C. castellanoides subsp. talaverae y C. castellanoides subsp. arundana. Además, para C. castellana Boiss. \& Reuter se propone el binomen prioritario $C$. aristata Hoffmanns. \& Link.
\end{abstract}

Palabras clave. Asteraceae, Centaurea, corología, taxonomía, Península Ibérica.

ABSTRACT. Taxonomical notes on the genus Centaurea at the Iberian Peninsula. II. C. castellanoides Talavera and C. aristata Hoffmanns. \& Link (Asteraceae). Morphologic variability of C. castellanoides Talavera an endemic species to the Iberian Peninsula is commented and three subspecies are recognized, two of them as new: C. castellanoides subsp. talaverae and C. castellanoides subsp. arundana. Also, C. aristata Hoffmanns. \& Link is proposed as the correct name for C. castellana Boiss. \& Reuter.

Key words. Asteraceae, Centaurea, chorology, taxonomy, Iberian Peninsula.

\section{INTRODUCCIÓN}

El género Centaurea L., que comprende unas 250 especies a nivel mundial (Susanna
\& Garcia-Jacas, 2007), distribuidas sobre todo por la Región Mediterránea y el SW de Asia, está siendo revisado taxonómicamente para la obra Flora Iberica (ed. S. 
Castroviejo). Se trata de uno de los géneros más diversificados y con mayor número de endemismos en la Península Ibérica, con no menos de un centenar de táxones (Dostál, 1976). Por ello, su estudio está siendo llevado a cabo por partes, abordando los grupos infragenéricos tradicionalmente reconocidos, a sabiendas no obstante de que muchos de ellos no tienen respaldo en la actualidad (Gabrielian, 1995; Susanna et al., 1995; Susanna \& Garcia-Jacas, 2007; Garcia-Jacas et al., 2000 y 2001; Font et al., 2002; Garcia-Jacas et al., 2006; SuárezSantiago, Salinas et al., 2007; SuárezSantiago, Blanca et al., 2007, etc.). Se trata de una opción que facilita el estudio por parte de diversos especialistas.

Recientemente, y con este objetivo, se ha efectuado una revisión para la Península Ibérica (López, 2008) del grupo de $C$. paniculata L. (Centaurea L. sect. Centaurea), de la que han derivado muchas novedades taxonómicas y corológicas respecto de los antecedentes previos disponibles (Arènes, 1949 \& 1951; Dostál, 1976). Algunas de ellas, así como la clave para el grupo, han sido publicadas (López \& Devesa, 2008a), dando comienzo así una serie de la que la presente contribución constituye la segunda entrega.

Se comenta en esta contribución la variabilidad en el territorio de $C$. castellanoides Talavera, un endemismo peninsular para el que se reconocen tres subespecies, claramente delimitadas por caracteres morfológicos y con respaldo corológico, dos de ellas nuevas: $C$. castellanoides subsp. talaverae y $C$. castellanoides subsp. arundana. Además, para C. castellana Boiss. \& Reuter, un endemismo del $\mathrm{C}$ de España con notables afinidades morfológicas con el anterior, se propone el binomen prioritario $C$. aristata Hoffmanns. \& Link.

\section{MATERIAL Y MÉTODOS}

El estudio morfológico y biométrico en el que se fundamente esta contribución ha sido llevado a cabo en material recolectado por los autores y en el conservado en diferentes herbarios (BC, BCN, COFC, COI, COI-Willk., JACA, JAEN, LISE, LOU, MA, MAF, SALA, SEV, UNEX), algunos de los cuales contienen material de interés para las lectotipificaciones, aunque otros han sido consultados exclusivamente a estos efectos (G).

\section{RESULTADOS Y DISCUSIÓN}

C. castellanoides Talavera in Lagascalia 12: 271 (1984)

C. paniculata var. baetica Pau in Carta Bot. 3: 5 (1906), non C. baetica (Willk.) Pau in Mem. Mus. Ci. Nat. Barcelona, Ser. Bot. 1(3): 21 (1925)

C. castellana var. baetica (Pau) Cuatrec. in Trab. Mus. Ci. Nat. Barcelona 12: 468 (1929)

C. micrantha var. baetica (Pau) Pau in Trab. Mus. Ci. Nat. Barcelona 12: 468 (1929)

Holotypus: "Elisee Reverchon. Plantes D'Espagne 1905 / (Province de Jaen) / N ${ }^{\circ}$ 409 / Centaurea castellana Boiss. / Le Pozo; lieux arides et calcaires, / 1500. métres. Juillet" (MA 135264; Talavera, 1984).

Distribución general: endemismo del S y E de la Península Ibérica. Esp.: Ab B Ca Co CR Cu Gr Gu J M Ma Mu So Te To Z (fig. 1).

Hábitat: claros y orlas de bosques principalmente encinares y pinares-, matorrales, cunetas y bordes de caminos, taludes y baldíos, por lo general sobre suelos pedregosos o arenosos, de naturaleza calcárea, no excesivamente nitrificados; (270)536-1.700 m. 


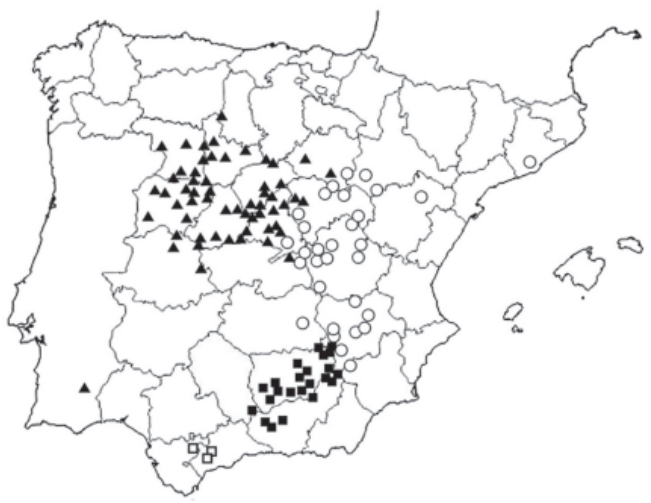

Figura 1. Área de distribución de: $\bullet$ : Centaurea aristata; : Centaurea castellanoides subsp. castellanoides; O: Centaurea castellanoides subsp. talaverae; $\square$ : Centaurea castellanoides subsp. arundana.

Observaciones: De todos los táxones del grupo $C$. paniculata con representación en el territorio, C. cordubensis Font Quer, $C$. bethurica E. López \& Devesa y $C$. castellanoides son los únicos que presentan aquenios más o menos obovoide-obcónicos y ennegrecidos en la madurez, pero mientras que los dos primeros presenta el apéndice de las brácteas involucrales con una espina de 1,5-4(5) $\mathrm{mm}$, claramente mayor que las fimbrias laterales, en el tercero culmina en un mucrón o arista de 0,3-1,5 mm, menor que estas (López \& Devesa, 2008a). Además, $C$. cordubensis y $C$. bethurica habitan en el cuadrante SW de la Península, con claras apetencias por los substratos silíceos, mientras que $C$. castellanoides es calcícola y su área se extiende por el S y E de España.

De acuerdo con Talavera (1984), la especie guarda gran similitud morfológica con C. castellana Boiss. \& Reuter (véase más adelante), pero a diferencia de esta, según indica, el apéndice de las brácteas involucrales medias carece de una membrana decurrente hialina, además de poseer una espina apical más pequeña. El estudio de gran cantidad de material de $C$. castellanoides confirma la observación de Talavera, pero en poblaciones alejadas del ámbito geográfico donde fue descrita la especie, existen dos grupos de poblaciones que sí presentan este carácter. Por un lado, el de las plantas de las sierras de Grazalema y Ronda, con involucro de 10-12 x 4-7 mm, redondeado en la base, y por otro el de las plantas del E-SE de España, de capítulos algo menores e involucro de 7,5-10 x 2-4 mm, atenuado en la base, dos patrones de variación que se describen aquí con categoría de subespecie: C. castellanoides subsp. arundana y $C$. castellanoides subsp. talaverae.

\section{Clave para las subespecies}

1. Apéndice de las brácteas involucrales medias no decurrente en una membrana hialina ........ a. subsp. castellanoides

1. Apéndice de las brácteas involucrales medias decurrente en una corta membrana hialina ... 2

2. Involucro 10-12 x 4-7 mm, redondeado en la base .......................... b. subsp. arundana

2. Involucro 7,5-10 x 2-4 mm, atenuado en la base c. subsp. talaverae

\section{a. subsp. castellanoides}

C. paniculata subvar. escobilla Coss. ex Arènes in Agron. Lusit. 11(1): 19 (1949) [Ind. loc.: "Portugal: Trás-os-Montes, Beira Baixa. Espagne: Aragon, Nouvelle Castille, Murcie, Andalousie, Grenade", "Bourgeau, Pl. Esp. 1001 (1850)"; Lectotypus: non vidi]

C. escobilla Coss. in Bourg., Pl. Espagne: 1001 (1850), in schaed., nom. nudum

C. escobilla Coss. in Notes Pl. Crit. 115 (1851), nom. inval., pro syn.

C. escobilla Coss. ex Nyman, Consp. Fl. Eur. 426 (1879), nom. inval., pro syn. 
C. paniculata var. cossoniana Arènes in Mem. Mus. Natl. Hist. Nat. Paris, Sér. B, Bot. 1(2): 200 (1951), non C. cossoniana Ball. in J. Bot. 11: 369 (1873) [Ind. loc.: "Espagne. - Jaen, Sierra de Segura; Bourgeau, Pl. Esp. (1850) sans $n^{\circ}$ pp., sub: C. C. Escobilla Coss. Var. (Coss.)"; Lectotypus: non vidi]

C. paniculata subsp. cossoniana (Arènes) Dostál in Bot. J. Linn. Soc. 71: 199 (1976)

Tallos 30-63 cm. Capítulos solitarios, con involucro 8-12 x 2,5-4 mm, estrechamente ovoide-cilíndrico, atenuado en la base; apéndice de las brácteas involucrales medias 1,2-2,6 mm, pectinado-fimbriado, de color pardo o pardo amarillento, erecto o erectopatente, glabro, no decurrente en una membrana hialina, con mucrón o arista apical de 0,3-1,4 $\mathrm{mm}$ y fimbrias laterales de 0,61,3 mm, en número de 3-7 a cada lado. Aquenios 3-4,2 $\mathrm{mm}$, negruzcos en la madurez. Vilano 0,7-1,6 mm, menor de la mitad del tamaño del aquenio. Florece de junio a septiembre.

Número cromosómico: $2 n=18$ (López \& Devesa, 2008b).

Distribución en el territorio: S \& SE de la Península Ibérica. Esp.: Ab Co Gr J (fig. 1).

Hábitat: En claros y orlas de encinares y matorrales, cunetas y bordes de caminos, taludes y baldíos, por lo general sobre suelos pedregosos o de textura gruesa, de naturaleza calcárea, no excesivamente nitrificados. 620 $1.700 \mathrm{~m}$.

b. subsp. arundana E. López \& Devesa, subsp. nova (fig. 2)

Caules $15-60 \mathrm{~cm}$. Capitula solitaria, quod ad involucrum attinet 10-12 × 4-7 mm, ovoideo-cylindrica, basi rotundata; eorum bractearum involucralium mediarum appendix 1,5-2,8 mm, fusca vel fusciatrata, erecta, lanuginosa vel glabra, in brevem hyalinam membranam decurrens, apicali mucrone aut arista 0,3-1,2 mm longis praedita pectinatisque fimbriis lateralibus 0,8-1,9 mm utrinque 4-7. Achaenia 2,7-4,3 $\mathrm{mm}$, matura nigricantia. Pappus 1,3-2,5 mm (achaenium aequans fere aut brevior satis). Floret a Iunio usque ad Augustum.

Holotypus: "Centaurea paniculata/var. baetica Pau / España. Málaga: Parque Natural Sierra de las Nieves. / A $1 \mathrm{~km}$ de la entrada por ctra. Ronda - / San Pedro de Alcántara. / En encinar basófilo / 10.7.2004 / leg: E. López \& F.J. Valtueña / det: E. López” (UNEX 34477).

Tallos 15-60 cm. Capítulos solitarios, con involucro $10-12 \times 4-7 \mathrm{~mm}$, ovoidecilíndrico, redondeado en la base; apéndice de las brácteas involucrales medias 1,5-2,8 $\mathrm{mm}$, pectinado-fimbriado, de color pardo a pardo obscuro, erecto, glabro o lanuginoso, decurrente en una corta membrana hialina, con mucrón o arista apical de 0,3-1,2 mm y fimbrias laterales de 0,8-1,9 $\mathrm{mm}$, en número de 4-7 a cada lado. Aquenios 2,7-4,3 mm, negruzcos en la madurez. Vilano 1,3-2,5 $\mathrm{mm}$, igual a la mitad del aquenio o más pequeño. Florece de junio a agosto.

Número cromosómico: $2 n=18$ (López \& Devesa, 2008b).

Distribución en el territorio: $\mathrm{S}$ de la Península Ibérica (sierras de Grazalema y Ronda). Esp.: Ca Ma (fig. 1).

Hábitat: En claros y orlas de bosques principalmente encinares y pinares-, matorrales, cunetas y bordes de caminos, taludes y baldíos, por lo general sobre suelos pedregosos o de textura gruesa, de naturaleza calcárea, no excesivamente nitrificados. 700-1.500 m.

c. subsp. talaverae E. López \& Devesa, subsp. nova

C. gracillima Pau in schaed (MA 135252), nom. nudum, non C. gracillima 


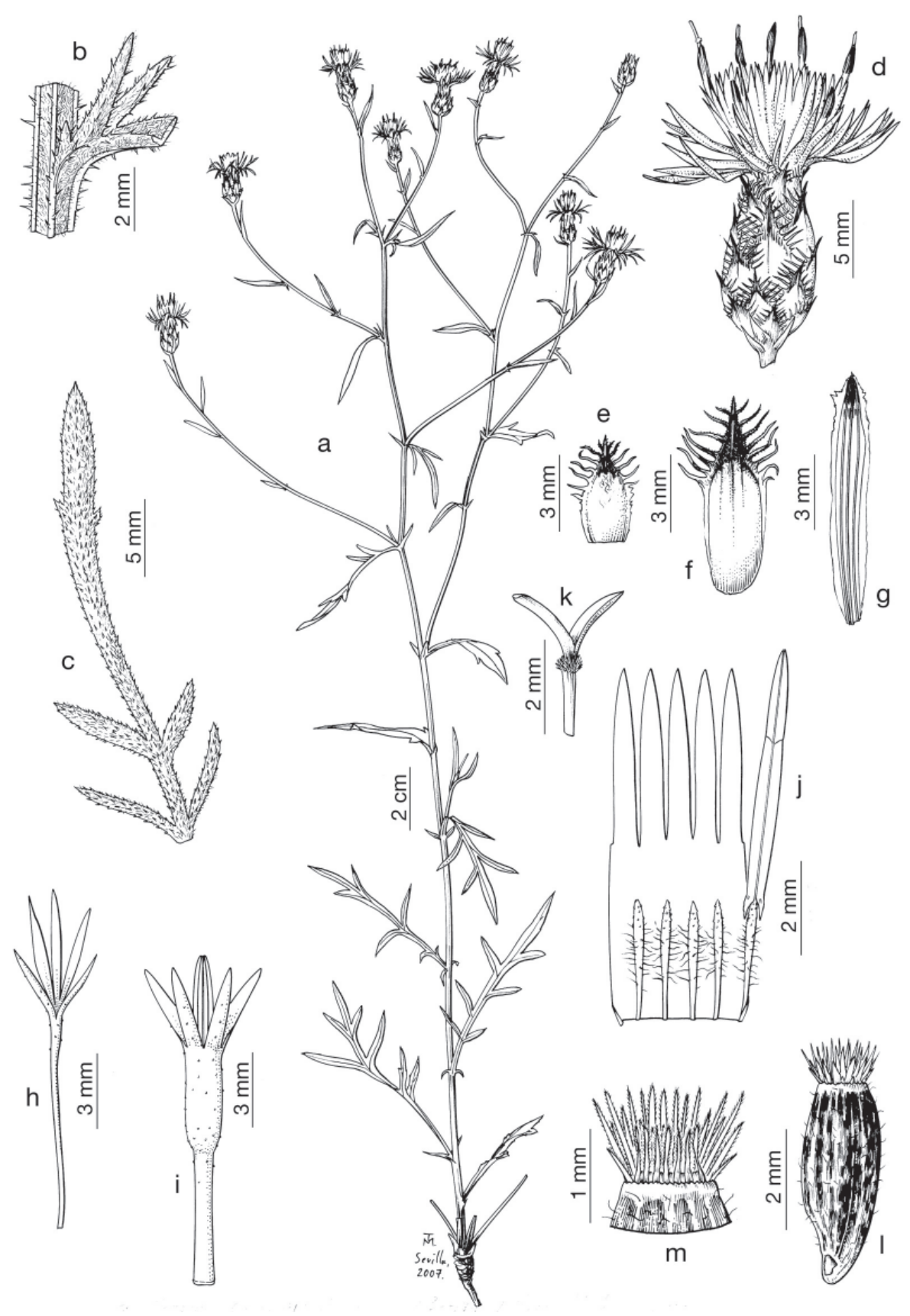

Figura 2. Centaurea castellanoides subsp. arundana. A, porte; B, nudo; C, hoja basal; D, capítulo; E, bráctea externa del involucro; F, bráctea media del involucro; $\mathrm{G}$, bráctea interna del involucro; $\mathrm{H}$, flor externa estéril; I, flor interna fértil; J, corola de flor interna fértil seccionada mostrando los filamentos estaminales y una antera; K, parte superior del estilo y ramas estigmáticas; L, aquenio; M, vilano mostrando el verticilo interno. 
Wagenitz in Notes Roy. Bot. Gard. Edinburgh 33(2): 225 (1974)

Caules 13-61 cm. Capitula solitaria vel nonnumquam geminata, quod ad involucrum attinet 7,5-10 × 2-4 mm, ovoideo-cylindrica, basi attenuata; eorum bractearum involucralium mediarum appendix 1-3 mm, fusca, erecta vel erecto-patens, glabra, in brevem hyalinam membranam decurrens, apicali mucrone aut arista -0,3-1,5 mm longis - praedita pectinatisque fimbriis lateralibus 0,6-2 $\mathrm{mm}$-utrinque 3-6-. Achaenia 2,3-3,5 mm, matura nigricantia. Pappus 0,5-1,5 (infra dimidium longitudinis achaenii). Floret a Iunio usque ad Septembrem.

Holotypus: "Centaurea castellana Boiss. \& Reuter / España. Toledo: Noblejas. / Carretera dirección a Dos Barrios. / Taludes calizos. / 7.7.2005 / leg: E. López / det: E. López 70/05” (UNEX 34478).

Tallos 13-61 cm. Capítulos solitarios o a veces en parejas, con involucro 7,5-10 x 2-4 mm, ovoide-cilíndrico, atenuado en la base; apéndice de las brácteas involucrales medias 1-3 mm, pectinado-fimbriado, de color pardo, erecto o erecto-patente, glabro, decurrente en una corta membrana hialina, con mucrón o arista apical de 0,3-1,5 mm y fimbrias laterales de $0,6-2 \mathrm{~mm}$, en número de 3-6 a cada lado. Aquenios 2,3-3,5 mm, negruzcos en la madurez. Vilano $0,5-1,5$ $\mathrm{mm}$, más pequeño que la mitad del aquenio. Florece de junio a septiembre.

Número cromosómico: $2 n=18$ (López \& Devesa, 2008b).

Distribución en el territorio: SE y CE de la Península Ibérica. Esp.: Ab B CR Cu $\mathrm{Gu} \mathrm{M} \mathrm{Mu}$ So Te To Z (fig. 1).

Hábitat: En claros y orlas de bosques principalmente pinares-, matorrales, cunetas y bordes de caminos, taludes y baldíos, por lo general sobre suelos pedregosos o arenosos, de naturaleza calcárea, no excesivamente nitrificados. (270) 536-1200 m.
Observaciones. - No son infrecuentes en las zonas de contacto plantas con características intermedias entre esta subespecie y C. aristata Hoffmanns. \& Link

C. aristata Hoffmanns. \& Link, Fl. Portug. 2: $226(1820-1828)$

C. paniculata subsp. aristata (Hoffmanns.\& Link) Cout., Fl. Portugal: 657 (1913)

Acosta aristata (Hoffmans. \& Link) Holub in Preslia 46: 226 (1974)

C. castellana Boiss. \& Reut. in Boiss., Diagn. Pl. Orient. ser. 1, 6: 129 (1846) [Ind. loc.: "Hab. in Castellâ Novà, provincia la Mancha ubique Boiss. Reut.". Lectotypus: "Centaurea paniculata (Matr Castellana) [sic.] Boiss./ et Reut./ var!/ San Bernardino/ 25 jul. 1841" [G 00074172-1; Burdet et al., 1983)]

C. paniculata var. castellana (Boiss. \& Reut.) Gugler in Ann. Hist.-Nat. Mus. Natl. Hung. 6: 158 (1907)

C. paniculata var. castellana (Boiss. \& Reut.) Cout., Fl. Portugal: 657 (1913), comb. superfl.

C. paniculata subvar. eucastellana Arènes in Agron. Lusit. 11(1): 19 (1949), nom. inval.

C. paniculata subsp. castellana (Boiss. \& Reut.) Dostál in Bot. J. Linn. Soc. 71: 199 (1976)

C. castellana var. asperrima Sennen in Pl. Espagne 1929: 70 (1936) [Ind. loc: "Madrid: Dehesa de la Villa, coteaux, 680 m.". Lectotypus: "1929. Plantes D'Espagne. - F. Sennen / no 7163 / Centaurea Castellana Boiss. Reut. / var. asperrima Sennen / Madrid, à la Dehesa de la Villa, coteaux, 680 m. / 1929-20-VII Leg. Sennen et Jeronimo / Note.- Nous avons trouvé quelques pieds C. ornata x Castellana/ = C. Villaris Sennen." (BC-Sennen $\mathrm{s} / \mathrm{n}$; se selecciona como 


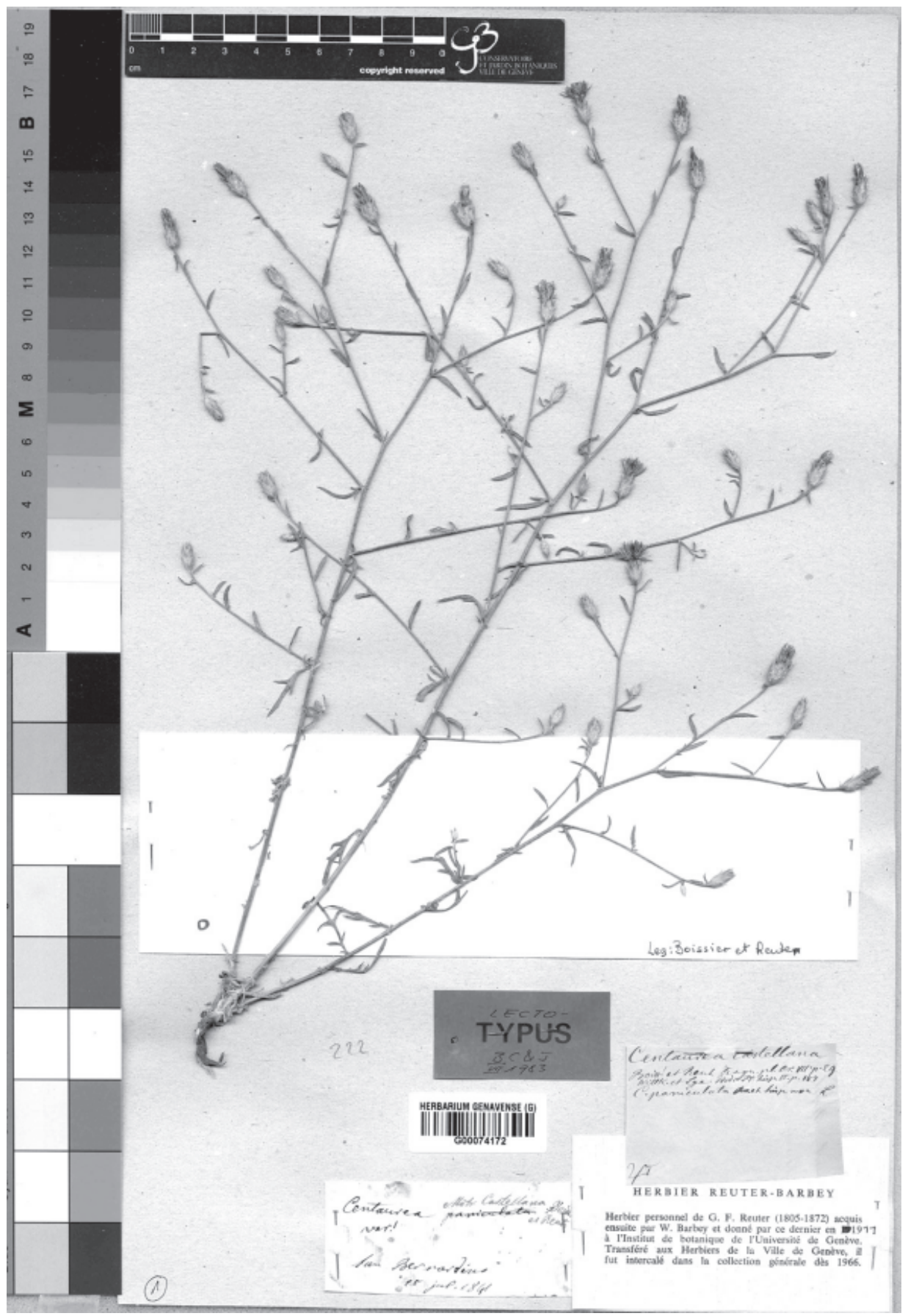

Figura 3. Lectotypus de C. castellana Boiss. \& Reut. (G 00074172-1). 
lectótipo el único fragmento contenido en el pliego; Isotypus: BC 84554, BCN s/n, MA 135243].

Ind. loc.: "Près de Mertola sur le bord de la Guadiane"

Número cromosómico: $2 n=36$ (López \& Devesa, 2008b).

Distribución general: C y CW la Península Ibérica, muy rara en el SW. Esp.: Av Cc Gu M P Sa Sg So To Va Za. Port.: BAl. (fig. 1).

Hábitat: En claros y orlas de bosques principalmente encinares, melojares y pinares-, matorrales -sobre todo tomillares y escobonales-, cunetas y bordes de caminos, taludes y baldíos, por lo general sobre suelos pedregosos o arenosos, tanto de naturaleza calcárea como silícea, no excesivamente nitrificados; 100-1.290 m.

Observaciones: Aunque por razones conocidas no es posible estudiar el material tipo de C. aristata Hoffmanns. \& Link, sí se ha visto material relativamente reciente procedente de la localidad típica (c. Mértola, Baixo Alentejo) y que se corresponde sin duda alguna con la descripción, y cuyas características se corresponden también con las de C. castellana Boiss. \& Reuter, lo que ya apuntaron Sampaio (1913) y Franco (1984).

Al estudiar el material tipo de Boissier \& Reuter, se está de acuerdo con la lectotipificación efectuada por Burdet et al. (1983, fig. 3) para C. castellana, y en los isótipos designados ( $\mathrm{G} 00074171$ y $\mathrm{G}$ 00074172-2), que proceden de la misma localidad y posiblemente de la misma recolección. Sin embargo, la identificación del síntipo procedente de "rochers de/ Pto. Lapiche" (G 00074173) es errónea, tratándose de un claro ejemplar de $C$. cordubensis (López \& Devesa, 2008 a).

Finalmente, señalar que $C$. aristata coexiste en algunas zonas del C de España con otros táxones del mismo grupo o más o menos afines, pudiéndose encontrar en las zonas de contacto plantas con características intermedias. Se han encontrado plantas con esta peculiaridad en las provincias de Guadalajara y Toledo, transicionales hacia C. castellanoides subsp. talaverae [ESPAÑA. Guadalajara. La Fuensaviñán, 14-VII-1983, Monge (SALA 44693). Toledo. Término de Ocaña, 21-VI-1965, Rivas Goday \& Borja (BCN s/n, MA 204569, MA 239892, MAF 67988, MAF 97941 \& SEV 5256)]; en las de Cáceres y Toledo, intermedias con $C$. bethurica [ESPAÑA. Cáceres. Pr. Mirabel, 5-VII-1924, Gros (JAEN 644480 \& SEV 204061). Toledo. La Nava de Ricomalilla, 7-VII-1977, Segura (SEV 100956)], y también con C. alba L. en las regiones más orientales del Sistema Central.

\section{ANEXO}

\section{Material estudiado}

Centaurea castellanoides subsp. castellanoides ESPAÑA. Albacete. Alcaraz, sin fecha, Fernández Casas (MA 408035). Calar del Mundo, 6-IX-1950, Rivas Goday \& Monasterio (MAF 87690). Villaverde de Guadalimar, 16VIII-2002, Arán \& Tohá (MA 703724). Córdoba. Priego de Córdoba, 4-VII-1980, Muñoz \& Tormo (SEV 123844); ídem, Sierra de Horconera, 4VIII-1980, Muñoz et al. (MA 394171 \& SALA 28835). Granada. Carretera entre Íllora y Montefrío, 30-VII-2004, E. López (UNEX 34476); ídem, 8-VI-2006, E. López (COFC 30736). Huéscar, Cortijo La Losa, 19-VII-1985, J. M. \& G. Montserrat (JACA 194986). Pr. Montefrío, 24-VII-1984, Quesada et al. (MA 416748). S ${ }^{a}$ de Alfacar, de "El Jardín" de la Fuente de la Teja, VIII-1979, J. M. Martínez (BC 632034); ídem, Alfaguara, 14-X-1980, F. Pérez \& Molero (JACA R280363). Sierra Sagra, cruce carretera Puebla a la Losa con la Santas, 8-X1978, sin recolector (MA 229536). Jaén. Barrancón de Valentina [barranco del río 
Guadalentín], VII, Reverchon (MA 135265). Campillo de Arenas, C. Quemado, 11-VII-1977, Fernández López (JAEN 772060); ídem, 29-VII1992, Fernández et al. (JAEN 922555). Ctra. Jaén-Granada, sin fecha, Fernández Morales (MA 329215). Ctra. Jaén-Los Villares, 16-X-1973, Fernández López (JAEN 73858). Cazorla, 25VIII-1945, E.B. (MA 201218); ídem, 22-IX-1975, González Rebollar et al. (MA 481415); ídem, barranco del río Guadalentín, 19-VIII-1976, González Rebollar et al. (MA 481413). El Moralico de Genave, 8-X-1978, P. Montserrat (JACA 440478). Fuensanta, 21-VII-1925, Cuatrecasas (MA 135263). Grajales, 17-VII1992, Fernández López (JAEN 922147). Hacia La Moraleda, 26-VII-1925, Cuatrecasas (BC 34795 \& MAF 10334). Jaén-Jabalcuz, 24-VI1976, Fernández López (JAEN 761371). La Iruela, El Cantalar, 27-IX-1992, Fernández López (JAEN 920985). La Pozo [E1 Pozo], VII, Reverchon (MA 135264). Los Villares, La Pandera, sin fecha, Fernández López (JAEN 741772); ídem, Los Cañones, 23-IX-1973, Fernández López (JAEN 73693). Martos, Sierra de la Grana, 31-VII-1992, Fernández López et al. (JAEN 922638). Noalejo, 8-VII-1976, Fernández López (JAEN 761507). Orcera, Puente Picorzo, 8-VIII-1996, Fernández Ocaña \& Fernández Prieto (JAEN 961271). Pontones, 24VII-1975, González Rebollar et al. (MA 481414). Pte. La Sierra, Silla de la Reina, 15-VI-1976, Fernández López (JAEN 761276). Quesada, 2XI-1956, Galiano (MA 190327). Santiago de la Espada, 17-VII-1975, González Rebollar et al. (MA 481416). Santisteban del Puerto, Cerro de S. Marcos, 4-VI-1995, J. L. Hervás (JAEN 956016). Segura de la Sierra, 18-VII-1986, Pajarón (MA 508198). Sierra de Cazorla, 5-III1980, G. López et al. (MA 216055). Sierra de Segura, 12-IX-1954, Borja et al. (MA 190326). Valdepeñas de Jaén a Frailes, 30-VI-1982, C. Fernández (JAEN 821314 \& MA 716211); ídem, a $7 \mathrm{~km}$ de Chircales, 12-X-1992, Peinado (JAEN 921064); ídem, Ventisqueros, 19-VII-1995, Torres Cordero (JAEN 991393). Villacarrillo, Fuente Higuera, 29-VI-1979, Fernández López (JAEN 791194); ídem, Pantano de Aguascebas, 17-VII-1995, Fernández López (JAEN 950873). Villanueva del Arzobispo, 13-X-1978, P. Montserrat (JACA 452878).

\section{Centaurea castellanoides subsp. arundana}

ESPAÑA. Cádiz. Grazalema, 15-VII-1930, Ceballos \& C. Vicioso (MA 135204); ídem, Cerro San Cristóbal, 2-VII-1983, Aparicio (MA 490980 \& SEV 208978); ídem, Lomas de Higueroba, 14VII-1925, Font Quer (BC 91465), ídem, Sierra del Pinar, 17-VII-1984, Aparicio \& F.G. Martín (MA 461890). Málaga. Ronda, 6-VI-1987, Montilla (SEV 208934). Sierra de las Nieves, 10-VII-2004, E. López \& Valtueña (UNEX 34477); ídem, 11 \& 12-VII-1919, Gros (MA 135203); ídem, 20-VII-1925, Gros (BC 91466); ídem, 20-VII-1925, Font Quer \& Gros (JAEN 644497, MA 702562 \& MAF 163479); ídem, pr. Ronda, 12-VII-1919, Gros (BC 34798). Sierra de Ronda, 28-VII, Reverchon (MA 135262).

\section{Centaurea castellanoides subsp. talaverae}

ESPAÑA. Albacete. Chinchilla \& Chamartín, 19 \& 20-VII-1920, Sennen \& Jerónimo (BC-Sennen $\mathrm{s} / \mathrm{n}, \mathrm{BCN} \mathrm{s} / \mathrm{n} \& \mathrm{MA}$ 135258). Carretera Albacete y Albadalejo, 6-III1980, Valdés et al. (MA 216056). Carretera entre la Hoz y Alcaraz, 30-VII-2004, E. López (UNEX 35212); ídem, 10-VII-2003, A. Ortega \& J. López (COFC 30735). Entre Riópar y Elche de la Sierra, 9-VII-1982, Mateo \& Aguilella (MA 421496). Fuensanta, 21-VII-1925, Cuatrecasas (BC 34792 \& BC 34793). Pozo Hondo, 13-IX-2005, Aedo (MA 731899). San Pedro, 8-VII-1934, González Albo (MA 134405). Barcelona. Tarrasa, VI-1908, Cadevall (MA 135217). Ciudad Real. Sierra Alhambra, 1936, González Albo (MA 134406). Cuenca. Alcázar del Rey, 17-VII-1974, G. López (MA 444293). Alrededores de Solán de Cabras, 2-IX-1941, Caballero (MA 135248). Arcas, Los Gamellones, 10-VII-1999, Arán \& Tohá (MA 631746 \& MAF 157515). Cerca de Tarancón, Las Tinajas, 21-VII-1977, G. López (MA 452495). Hoz de Beteta, 4-VII-1974, Valdés \& G. López (MA 446642); ídem, 4-VIII-1974, Valdés (MAF 137292). Huete, 12-VII-1976, M. Costa (MA 504399). Millanes, 12-VII-1976, M. Costa (MA 504397 \& MA 504412). Monte de Palancares, 22X-1921, Huguet del Villar (MAF 56901). Pinares de El Tobar, 20-VIII-1934, Caballero (MA 135249). Puente Vadillos, VII-1932, Caballero (MA 135250). Saceda-Trasierra, Sierra de Altomira, 26-VI-1999, Arán \& Tohá (MA 631747). Salida hacia Uña, Hoz del Júcar, 22- 
IX-1974, Bondía et al. (MA 442061). Uclés, sin fecha, Colmeiro (MA 135251). Vega del río Tovar, 8-VII-1934, González Albo (MA 134404). Guadalajara. Ciruelos, 20-VII-1976, Casaseca (SALA 10367). Guadalajara, VII-1904, Mas y Guindal (MAF 62928). La Alcarria, subida a Aranzueque, 21-XI-1969, Rivas Goday \& Rivas Martínez (MAF 90902 \& SALA 64424). Loranca de Tajuña, VII-1921, Del Coto (MAF 10336). Sigüenza, 10-IX-1949, Rivas Goday \& Galiano (MAF 10335 \& MAF 76978). Sigüenza, 9-VIII1962, Galiano (MA 239587 \& MAF 111927); ídem, 12-VII-1906, B. Vicioso (MA 135254). Terzaga, 31-VII-1985, Mateo (MA 313547). Madrid. Morata de Tajuña, VIII-1919, C. Vicioso (MA 135238). Murcia. El Sabinar, 7-VIII-1992, De la Torre (JACA 348193). Soria. Carretera Soria-Medinaceli, 26-VII-1988, Pedrol (MA 459576). Jubera, 17-VIII-1991, Segura (SEV 208941). Monteagudo de las Vicarias, 1-VIII1994, Martínez (JACA 310094). Teruel. Andorra, 28-VII-1872, Loscos (COI-Willk. s/n). Toledo. Noblejas, 7-VII-05, E. López (UNEX 34478). Zaragoza. Calatayud, 26-VII-1897, C. Vicioso (MA 135247); ídem, 26-VII-1897, B. Vicioso (MA 135252); ídem, VII-1897, Mas y Guindal (MAF 63300). Daroca, VII-1850, Wilkomm (COI-Willk. s/n \& MA 177034); ídem, VII-1908, B. Vicioso (MA 135253).

\section{Centaurea aristata}

ESPAÑA. Ávila. Ávila, VII-1964, Bellot \& Borja (MA 199451, MA 199452, MA 201219, MA 201236, MAF 134060, SALA 599, SALA 26378, SALA 64944 \& SALA 83811). Cuevas del Valle, 20-VIII-1985, Segura (MA 579932, MA 580051, MA 580492 \& MA 586560). Monbeltrán, Vallejón, 3-VIII-1992, Segura (JACA 200796, MA 580258, MA 581100 \& SEV 208948). Navalperal de Pinares, 9-X-1979, Valdés et al. (MA 442843). Navas del Marqués, 16-VII-1970, Valdés (SALA 23595). Urraca Miguel, hacia Ojos Albos, 29-VIII-1982, Cantó \& D. Sánchez (MAF 123303). Cáceres. Baños de Montemayor, VIII1903, Escribano (MA 135259). Peraleda de San Román, 20-V-1967, Ladero (MAF 80798). Plasencia, 28-VI-1956, Serres (MA 164446). Talaveruela, 19-VII-1989, Amor (SALA 84721). Guadalajara. Hita, IX-1969, Ron (MA 196847).
Puebla de Vallés, 8-VII-1981, De la Fuente (MAF 130712). Madrid. Batres, 4-VII-1977, Borja (MAF 152350). Casa de Campo, VII-1892, sin recolector (MAF 63295). Chamartín de la Rosa, VIII-1897, Pau (BC 34789); ídem, VIII-1912, C. Vicioso \& F. Beltrán (MA 135241 \& MA 722156); ídem, 14-VI-1924, R. M. (MAF 10340). Chinchilla \& Chamartín, 19 \& 20-VII-1920, Sennen \& Jerónimo (BC-Sennen s/n, BCN s/n \& MA 135258). Collado Mediano - El Escorial, VIII-1914, C. Vicioso (BC 37791). Collado Mediano, VIII-1912, C. Vicioso (MA 135234). Colmenar Viejo, sin fecha, sin recolector (MA 135213). De Cercedilla a Guadarrama, 18-VIII1912, C. Vicioso (LISE 46502, MA 179664 \& MA 201228). Dehesa de Batres, 10-VII-1975, Borja (SALA 64425). Dehesa de la Villa, 20-VII-1929, Sennen \& Jerónimo (BC 84554, BC-Sennen s/n, BCN s/n \& MA 135243); ídem, IX-1911, C. Vicioso (MA 135242); ídem, VIII-1913, $C$. Vicioso (MA 135242); ídem, VII \& VIII-1913, Beltrán (BC 34796, BC-Sennen s/n, MA 135240). El Paular, IX-1912, Isern (MA 136587). E1 Escorial, 22-VIII, Cutanda (MA 135236); ídem, VIII-1912, C. Vicioso (MA 135235); ídem, VII1964, Borja (MA 179663); ídem, VI-1920, Aterido (MA 144064); ídem, VIII-1921, R. M. (MAF 10341); ídem, VIII-1985, Izuzquiza (MA 401755). El Molar, 20-VI-1924, Font Quer \& Gros (JAEN 644494, MA 702564 \& MAF 163480); ídem, 20-VI-1924, Gros (BC 91408 \& MA 135239). Embalse de Santillana, 28-VI-1981, Sánchez-Mata (MAF 106494 \& SALA 67565). Encinar de la Parra, 22-VI-1964, P. Montserrat (JACA 210764). Entre Cercedilla y el Puerto de León, VIII-1912, C. Vicioso (MA 135232). Fuencarral, 28-VIII-1976, Pascual (MA 619801). Guadarrama, VII-1886, Lázaro (MAF 10339); ídem, VII-1890, Lázaro (LOU 3441). Inter Cercedilla et El Escorial, 17-IX., Isern (MA 135233). Madrid, 10-IX-1915, Jerónimo (BC 34785, BC-Sennen s/n, BCN s/n \& MA 135245); ídem, VII-1897, Pau (COI s/n); ídem, 13-VIII1897, Pau (MA 135244); ídem, VIII-1914, $C$. Vicioso (BC 34790). Pelayos de la Presa, 7-VII05, E. López (COFC 30739). Pozuelo de Alarcón, 13-VII-1838, García (MA 719792). Prox. Guadarrama, VI-1976, Llimona (BCN s/n). San Bernardino, 25-VII-1841, Boissier et al. (G 
00074171, G 00074172-1 \& G 00074172-2). San Martín de Valdeiglesias, 12-X., Cutanda (MA 135237); ídem, 7-VII-05, E. López (COFC 30686). Sierra de Guadarrama, VII-1964, Borja (MA 179665); ídem, VII-1960, Borja (MA 183478 \& MA 183480). Torrelodones, 14-VII1994, Gabriel y Galán (MA 571549). Villalba de Guadarrama, 15-VII-1951, De Bolòs (BC 115424). Palencia. Boadilla del Rioseco, 28-VI1950, Rivas Goday (MAF 87815). Salamanca. Aldealengua, 9-IX-1979, Rico (SALA 7356). Arapiles, 15-VII-1983, Fernández. Orcajo (SALA 33715). Balbilafuente, 2-VII-2003, Ortega \& E. López (COFC 30680). Ciperez, 1IX-1978, Amich (SALA 15183). Ciudad Rodrigo, 3-VII-1976, Rico (SALA 9606); ídem, VII-1964, Borja (MAF 104040). Cubo del Vino, 31-VII1981, Giráldez (SALA 31023). Guijuelo, 14-IX1963, P. Montserrat (JACA 156463). La Orbada, 3-X-1973, P. Montserrat (JACA 583873). Las Veguillas, 7-VII-1986, Monzón (SEV 208952). Palacios del Arzobispo, 27-VII-1976, J. Sánchez (SALA 17182). Paradinas de San Juan, Taberuela, 15-VIII-1966, F. González (JACA R280362). Pelabravo, 15-VII-1989, Pastor (SALA 57401). Peñaranda de Bracamonte, 10-VII-1968, Borja \& Mansanet (MAF 73876, MAF 74484 \& MAF 74485); ídem, 10-VII-1968, Borja (MA 228051); ídem, sin fecha, sin recolector $(\mathrm{BCN} \mathrm{s} / \mathrm{n})$; ídem, VII-1953, Losa (BCN s/n). Salamanca, VIII1850, Willkomm (COI-Willk. s/n). Traguntia, 14XI-1978, Amich (MA 228112 \& SALA 15184). Ventosa del Río Almar, 2-VII-1972, De Bolós (BC 608900). Segovia. El Espinar, VII-1943, Rivas Goday (MAF 78456). Espirdo, La Higuera, 14VIII-1988, Egido \& R. García (MA 739438); ídem, 20-VII-1989, Galán et al. (MA 739440). Palazuelos de Eresma, 29-VII-1896, R. García \& P. Egido (MA 739439); ídem, 16-IX-1896, R. García (MA 739441). Torreadrada, Pozarrón, 30VII-1984, Romero (MA 569069). Valtiendas, 30VII-1984, Romero (SALA 39571). Soria. Crisol, 15-VII-1990, Segura (MA 581992). El Burgo de Osma, 29-VII-2001, Molero (BCN s/n); ídem, Uxana, 19-VII-2001, Molero (BCN s/n). Torremediana, 21-VI-1991, Segura (JACA 200896 \& MA 580164). Toledo. De Navamorcuende a Sartajada, 7-VII-2005, E. López (UNEX 34479). Dosbarrios, sin fecha,
Laorga (MAF 121257). Montes de Toledo, VII1915, Cogolludo (MA 135256). Portillo de Toledo, VII, Cortés (MAF 10342). Sierra Alpuébrega, VII-1915, Cogolludo (MA 135257). Sortajada, 27-VIII-1990, Segura (MA 580276). Valladolid. Castromonte, 30-VI-1906, Sennen (BC-Sennen s/n). Castronuño, 2-VII-2003, Ortega \& E. López (COFC 30683 \& COFC 30689); ídem, 28-VIII-1988 (SALA 84263). Entre Mota del Marqués y Villardefrades, 28-VII1978, Talavera et al. (MA 211052, MAF 102108 \& SALA 13445). Santa Espina, sin fecha, Losa (MAF 10337). Villamarciel, 30-VII-1986, Ladero \& Valle (SALA 76914). Villanueva de los Infantes, 12-VII-2004, Devesa \& E. López (COFC 30682). Zamora. Abezames, Valcuervo, 2-VII-1990, García Ríos (SALA 53215). Carretera de los Alcañices, 25-VII-1939, Sierra (BC 91894). Cerecinos del Carrizal, Cuatrocaminos, 2-VIII-1990, García Rios (SALA 53218). Fuentesauco, 15-IX-1981, Giráldez (SALA 31020). Guimaré, 14-VII-1922, Luelmo (MA 135208). Morales de Toro, 22-V-1997, P. Montserrat et al. (JACA 114197). Vezdemarbán, Quiñones, 4-VIII-1990, García Ríos (SALA 53210); ídem, Valoria, 3-VII-1990, García Ríos (SALA 53216). Villanueva del Puente, 2-VII2003, Ortega \& E. López (COFC 30737)

PORTUGAL. Baixo Alentejo. Mertola, 13VI-1960, A. Fernandes et al. (COI 24239 \& MAF 75555).

SIN LOCALIDAD PRECISADA. Sin fecha, Cavanilles (MA 135246). Sin fecha, Borja (UNEX 306). Sin fecha, sin recolector (MA 229376). Sin fecha, sin recolector-Herb. Antiguo- (MA 149238). Sin fecha, Lagasca (MA 149241).

AGRADECIMIENTOS. El presente trabajo ha sido desarrollado en el contexto de la obra Flora Iberica, y financiado por los proyectos REN200204634-C05-04 y CGL2005-05471-C04-02, de la Dirección General de Investigación Científica y Técnica del Ministerio de Educación y Ciencia. Nuestro agradecimiento, igualmente, a todos los responsables de los herbarios e Instituciones citadas, y al Dr. Laínz por la traducción al latín de las diagnosis. Uno de los autores, E. López, estuvo adscrito como becario en formación (BES2003-1275) a cargo de los proyectos citados. 


\section{BIBLIOGRAFÍA}

ARÈNES, J. -1949- Les races lusitaniennes de Centaurea paniculata L. sensu latísimo. Agron. Lusit. 11: 5-32.

ARÈNES, J. -1951- Le groupe spécifique du Centaurea paniculata L. sensu latiss. Mém. Mus. Natl. Hist. Nat. Paris, Sér. B, Bot. 1 175-266.

BURDET, H. M., A. CHARPIN \& F. JACQUEMOUD -1983- Types nomenclateraux des taxa ibériques décrits par Boissier ou Reuter. IV. Cistacées à Composées. Candollea 38: 751802.

DOSTÁL, J. -1976- Centaurea L. In: Tutin, T.G., Heywood, V.H., Burges, N.A., Valentine, D.H., Walters, S.M. \& Webb, D.A. -eds.Flora Europaea 4: 254-301. Cambridge University Press. Cambridge.

FONT, M., T. GARNATJE, N. GARCIA-JACAS \& A. SUSANNA -2002- Delineation and phylogeny of Centaurea sect. Acrocentron based on DNA sequeces: a restoration of the genus Crocodylium and indirect evidence of introgression. Pl. Syst. Evol. 234: 15-26.

FRANCO, J.A. -1984- Nova flora de Portugal (Continente e Açores), 2. Lisboa.

GABRIELIAN, E. T. -1995- On the generic status of certain groups of Centaureinae (Compositae). In: Hind, D.J.N., Jeffrey, C. \& Pope, G.V. eds.- Advances in Compositae Systematics:145152. Royal Botanic Garden. Kew.

GARCIA-JACAS, N., A. SUSANA, V. MOZAFFARIAN \& R. ILARSAN -2000- The natural delimitation of Centaurea (Asteraceae: Cardueae): ITS sequence analysis of the Centaurea jacea group. Pl. Syst. Evol. 223: 185-199.

GARCIA-JACAS, N., A. SUSANNA, N. GARNATJE \& R. VILATERSANA -2001Generic Delimitation and Phylogeny of the Subtribe Centaureinae (Asteraceae): A Combined Nuclear and Chloroplast DNA Analysis. Ann. Bot. (Oxford) 87: 503-515.

GARCIA-JACAS, N., T. UYSAL, K. ROMASCHENKO, V. N. SUÁREZSANTIAGO, K. ERTUGRUL \& A. SUSANNA
-2006- Centaurea revisited: a molecular survey of the Jacea group. Ann. Bot. 98: 741-753.

LÓPEZ, E. -2008-Estudio taxonómico de Centaurea sect. Paniculatae (Hayek)Dostál $y$ sect. Phalolepis (Cass.) DC. En la Peninsula Ibérica. Tesis Doctoral. Universidad de Extremadura. Badajoz.

LÓPEZ, E. y J. A. DEVESA -2008a- Notas sobre el género Centaurea L. (Asteraceae) en la Península Ibérica. I. Centaurea cordubensis Font Quer, C. bethurica E. López \& Devesa, sp. nova. y C. schousboei Lange. Anales Jard. Bot. Madrid 65: XXX.

LÓPEZ, E. y J. A. DEVESA -2008b- Contribución al conocimiento cariológico del género Centaurea L. (Asteraceae) en la Península Ibérica. Acta Bot. Malacitana 33: 69-90.

SAMPAIO, G.A.S.F. -1913- Lista das espécies representadas no Herbário português. Oporto.

SUÁREZ SANTIAGO, V. N., M. J. SALINAS, N. GARCIA-JACAS, P. S. SOLTIS, D. E. SOLTIS \& G. BLANCA -2007- Reticulate evolution in the Acrolophus subgroup (Centaurea L., Compositae) from the western Mediterranean: origin and diversification of section Willkommia Blanca. Mol. Phyl. Evol. 43: 156-172.

SUÁREZ-SANTIAGO, V. N., G. BLANCA, M. RUÍZ-REJÓN \& M. A. GARRIDO-RAMOS -2007- Satellite-DNA evolutionary patterns under a complex evolutionary scenario: The case of Acrolophus subgroup (Centaurea L., Compositae) from the western Mediterranean. Gene 404: 80-92

SUSANNA, A., \& N. GARCIA-JACAS -2007Tribe Cardueae. In: Kadereit, J.W. \& Jeffrey, C. -eds.- The families and genera of vascular plants, 123-147. Springer.

SUSANNA, A., N. GARCIA-JACAS, D.E. SOLTIS \& P. S. SOLTIS -1995- Phylogenetic relationships in tribe Cardueae (Asteraceae) base on ITS sequences. Amer. J. Bot. 82: 1056-1068.

TALAVERA, S. -1984- Centaurea castellanoides Talavera. In: Notas Breves, $\mathrm{n}^{\circ} 131$. Lagascalia 12: 271-272. 